\title{
A Stochastic Crack Growth Model with Propagation Resistance as a Random Field ${ }^{*}$
}

\author{
Hiroshi Ishikawa ${ }^{a}$, Hiroaki Tanaka ${ }^{b}$ and Satoshi Wakasa ${ }^{c}$ \\ ${ }^{a}$ Dept. of Information Science, Kagawa University, Takamatsu, Kagawa 760 Japan \\ ${ }^{b}$ Dept. of Applied Mathematics \& Physics, Kyoto University, Kyoto 606-01 Japan \\ c Miura Institute of Research \& Development, Matsuyama, Ehime 799-26 Japan
}

A new stochastic crack growth model is proposed, in which the propagation resistance appearing in the empirically obtained crack growth law is mathematically modeled as a random field. First, a mathematical methodology to obtain the solution of the randomized crack growth equation is discussed. Next, an approximate method to obtain a probability distribution of the residual life is proposed. Finally, the method is applied to the random propagation problem of an edge crack under uniform tensile stressing, where numerical evaluation of the residual life distribution in this case is performed.

\section{Introduction}

In order to investigate the probability distribution of the fatigue crack propagation life due to the randomness caused by microscopic inhomogeneity of the material, it has been often the case to introduce a mathematical model based upon the well-known ParisErdogan crack growth law such that

$\frac{d a}{d n}=C_{0}(\Delta K)^{m}$

with $C_{0}$, the crack propagation resistance, being a variable of stochastic nature, and $m$ being a material constant. In the model developed by Tsurui and Ishikawa [1][2] (termed as TI-model) and other models proposed to date [3], the propagation resistance $C_{0}$ has been treated as a random process with temporal variation. That is, the growth equation (1) has been extended into a random differential equation, which is a differential equation with a temporally random input.

However, since the randomness induced in the actual materials will cause spatial random fluctuations in $C_{0}$, it is more appropriate, in reference to the engineering reality, to

*This research was supported in part by a Scientific Grant-In-Aid in 1993 (No. 05750066) from the Ministry of Education, Science and Culture, Japan. 
model $C_{0}$ as a random field than as a random process. Hence, Eq.(1) needs to be extended into the following random differential equation:

$\frac{d a}{d n}=\frac{C_{0}}{C(a)}(\Delta K)^{m} \quad\left(C_{0}:\right.$ const. $)$

where $C(a)$ is a random field showing a spatially random variation. The reciprocal form is selected so as to express the "resistance" clearly [4][5].

The random differential equation (2) is a differential equation defined on a random field, and it has a special feature that the variable to indicate the location of the random field is combined concurrently with a dependent variable of the equation. In this respect, a mathematical methodology to obtain the solution to the random differential equation in the form of Eq.(2) has been newly developed [6].

In this paper, according to a mathematical methodology developed by Tanaka and Tsurui [6] to define a solution for such a random differential equation of special type, we construct a new probabilistic model to obtain the probability distribution of the random growth of fatigue cracks. The result will be compared with TI-model through numerical computation of the residual life distribution, which plays a crucial role in the reliabilitybased design.

\section{Mathematical analysis of randomized crack growth equation}

In this section, we give a survey of the mathematical methodology first developed by Tanaka and Tsurui [6] to analyze the randomized crack growth equation (2).

\subsection{Definition of the solution}

For the sake of convenience in analysis, we use the dimensionless crack length $X=$ $a / a_{r}$, where $a_{r}$ represents the reference length. Suppose that the stress intensity factor range $\Delta K$ can be expressed as

$\Delta K=\alpha \Delta S_{0} \sqrt{X} f(X)$,

where $\Delta S_{0}$ is a stress amplitude (it is assumed to be constant), the function $f(\cdot)$ is a correcting function expressing the size effect of the component and $\alpha$ is a constant determined according to the geometry of the crack. Substituting Eq.(3) into Eq.(1), we can obtain

$\frac{d X}{d t}=\varepsilon g(X) \quad\left(g(X) \equiv\{\sqrt{X} f(X)\}^{m}\right)$,

in which we use a new time variable $t$ such that $\varepsilon a_{r} t=C_{0} \alpha^{m}\left(\Delta S_{0}\right)^{m} n$.

As mentioned in the preceding section, the microscopic inhomogeneity of the material gives rise to a spatially distributed random variation of the coefficient $\varepsilon$. Hence, we need to extend the differential equation (4) to the following random differential equation:

$\frac{d X}{d t}=\frac{\varepsilon}{C(X)} g(X)$,

where $C(x)$ is a random field. The solution of Eq.(5) will show a temporally random variation according to the spatially random variation of the random field $C(x)$, even if the initial value $X(0)=x_{0}$ is deterministic. 
If the material is macroscopically homogeneous, the random field $C(x)$ will be a homogeneous random field. That is, its first and second moments are given as

$\mathrm{E}[C(x)]=M_{C}($ const. $)$

$\mathrm{E}\left[\{C(x)-\mathrm{E}[C(x)]\}\left\{C\left(x+x^{\prime}\right)-\mathrm{E}\left[C\left(x+x^{\prime}\right)\right]\right\}\right]=\sigma_{C}^{2} \rho_{C}\left(x^{\prime}\right) \quad$ (independent of $x$ )

It should be noted that we can assume, without loss of generality, that the mean value $M_{C}$ takes on unity.

Integrating Eq.(5) formally under the initial condition $X(0)=x_{0}$, we have

$\int_{x_{0}}^{X(t)} \frac{C(x)}{g(x)} d x=\varepsilon t$.

It is natural to define the solution $X(t)$ of Eq.(5) as a function $X(t)$ satisfying the integral equation (8) provided that the left-hand side integral exists. However, if there is a nonzero probability that $C(x)$ takes a negative value, we can not uniquely define $X(t)$, since the left-hand side of Eq.(8) is not a monotonically increasing function. Such a situation should be taken into account even if the distribution range of $C(x)$ is restricted to the positive area, since we need to introduce the Markov approximation method [1], which is a kind of diffusion-approximation, in our practical analysis. Hence, introducing a new random field such that

$Z\left(x ; x_{0}\right) \equiv \int_{x_{0}}^{x} \frac{C\left(x^{\prime}\right)}{g\left(x^{\prime}\right)} d x^{\prime}$

we define the solution of Eq.(5), by making a partial revision on Eq.(8), as

$X(t) \equiv \inf _{x>x_{0}}\left\{Z\left(x ; x_{0}\right)=\varepsilon t\right\}$.

If the transformed random field $Z\left(x ; x_{0}\right)$ is almost certainly continuous, $X(t)$ can be uniquely defined with probability one.

Figure 1 gives a conceptual illustration of the definition of the solution $X(t)$, which is defined as a first passage location [7] of the random field $Z\left(x ; x_{0}\right)$ to a level $\varepsilon t$.

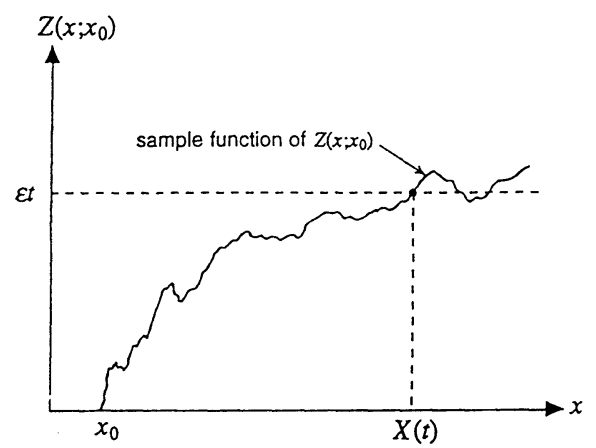

Fig.1 Relation between the solution $X(t)$ and the sample field of $Z\left(x ; x_{0}\right)$. 


\subsection{Death point}

If the function $g(x)$ rapidly diverges as $x \rightarrow \infty$, the transformed random field $Z\left(x ; x_{0}\right)$ may not diverge as $x \rightarrow \infty$ under the assumption that the random field $C(x)$ is homogeneous. In fact, if the exponent $m$ in the Paris law is greater than 2 , we have to take into account such a possibility [1].

At the time $t$ when $\varepsilon t$ is greater than the supremum of $Z\left(x ; x_{0}\right)$, it is natural to consider that the solution $X(t)$ has already diverged to infinity. Hence, for a given $t$, we define that the solution $X(t)$ lies in the state $\mathbf{D}$ if

$\sup _{x>x_{0}} Z\left(x ; x_{0}\right)<\varepsilon t$,

is satisfied. According to TI-model [1], we call the state $\mathbf{D}$ the death point.

\section{Probability distribution of the solution process and residual life distribution}

According to the definition of the solution $X(t)$ discussed in the preceding section, we need to solve a first passage problem for the transformed field $Z\left(x ; x_{0}\right)$ to obtain the probability distribution of the solution process $X(t)$. Unfortunately, however, since we do not have a complete methodology to solve a first passage problem of general type, it is very difficult to derive the exact probability distribution of the solution process $X(t)$. Hence, in this section, we give an approximate method [6] to derive the transition probability distribution of the solution $X(t)$ and the residual life distribution.

\subsection{Transition probability distribution of the solution process}

With the aid of Eqs.(6) and (7), we can obtain the mean and variance of the transformed field $Z\left(x ; x_{0}\right)$ as follows:

$$
\begin{aligned}
& \mu_{Z}\left(x ; x_{0}\right) \equiv \mathrm{E}\left[Z\left(x ; x_{0}\right)\right]=\int_{x_{0}}^{x} \frac{d x^{\prime}}{g\left(x^{\prime}\right)}, \\
& \sigma_{Z}^{2}\left(x ; x_{0}\right) \equiv \mathrm{E}\left[\left\{Z\left(x ; x_{0}\right)-\mathrm{E}\left[Z\left(x ; x_{0}\right)\right]\right\}^{2}\right]=\sigma_{C}^{2} \int_{x_{0}}^{x} \frac{d x^{\prime}}{g\left(x^{\prime}\right)} \int_{x_{0}}^{x} \frac{\rho_{C}\left(x^{\prime}-x^{\prime \prime}\right)}{g\left(x^{\prime \prime}\right)} d x^{\prime \prime} .
\end{aligned}
$$

If the random field $C(x)$ is a Gaussian field with independent increment, the transformed field $Z\left(x ; x_{0}\right)$ is also a Gaussian field, whose probabilistic properties can be completely specified by Eq.(12) and (13). Even under the situation in which $C(x)$ does not have such a property, we can treat $Z\left(x ; x_{0}\right)$ approximately as a Gaussian field by applying the Markov approximation method [1]. Hence, we can obtain an approximate form of the probability distribution function of $Z\left(x ; x_{0}\right)$ in the following form:

$P\left(z, x \mid x_{0}\right)=\operatorname{Pr}\left[Z\left(x ; x_{0}\right) \leq z\right]=\Phi\left[\frac{z-\mu_{Z}\left(x ; x_{0}\right)}{\sigma_{Z}\left(x ; x_{0}\right)}\right]$,

where $\Phi(\cdot)$ is the standardized normal distribution function.

It should be noted that the result gives a non-zero probability that $Z\left(x ; x_{0}\right)$ decreases as a function of $x$. However, we can almost neglect it if the spatial interval $\left|x-x_{0}\right|$ is sufficiently large enough in comparison to the spatial correlation distance of the random 
field $C(x)$. In this case, according to the definition given by Eq.(10), we can obtain an approximate relationship as

$W\left(x, t \mid x_{0},\right) \equiv \operatorname{Pr}\left[X(t) \leq x \mid X(0)=x_{0}\right] \simeq 1-\operatorname{Pr}\left[Z\left(x ; x_{0}\right) \leq \varepsilon t\right]$.

Hence, the transition probability distribution function of the solution process $X(t)$ is given as follows:

$W\left(x, t \mid x_{0}\right)=\Phi\left[\frac{\mu_{Z}\left(x ; x_{0}\right)-\varepsilon t}{\sigma_{Z}\left(x ; x_{0}\right)}\right]$,

which is a probability distribution of special type, whose domain is restricted to $x_{0}<x$. If the function $g(x)$ rapidly diverges as $x \rightarrow \infty, W\left(x, t \mid x_{0}\right)$ does not tend to unity as $x \rightarrow \infty$, since there is a non-zero probability that the solution $X(t)$ lies in the death point D.

\subsection{Residual life distribution}

Let $T\left(x_{0}, x_{c}\right)$ be a time when the sample function with the initial condition $X(0)=x_{0}$ arrives at a prescribed critical level $x_{c}\left(>x_{0}\right)$ for the first time. We call it the residual life of $X(t)$, and denote its probability distribution function as

$H\left(t \mid x_{0}, x_{c}\right) \equiv \operatorname{Pr}\left[T\left(x_{0}, x_{c}\right) \leq t\right]$.

According to Eq.(16), the probability that $X(t)$ decreases is exactly zero. Hence, we obtain

$H\left(t \mid x_{0}, x_{c}\right)=1-\operatorname{Pr}\left[X(t) \leq x_{c} \mid X(0)=x_{0}\right]=\bar{\Phi}\left[\frac{\mu_{Z}\left(x_{c} ; x_{0}\right)-\varepsilon t}{\sigma_{Z}\left(x_{c} ; x_{0}\right)}\right]$,

where $\bar{\Phi}(\cdot)$ is the complementary function of the standardized normal distribution. If the cracked component fails when the crack grows to the critical length $x_{c}, H\left(t \mid x_{0}, x_{c}\right)$ gives a probability of failure at time $t$. Thus, it must be kept at a very small value so as to make the component highly reliable in actual situations.

\subsection{Residual life distribution in TI model}

In TI-model, the random field $C(x)$ has been transformed into a random process reflecting a certain typical value of the solution as a function of time, that is, Eq.(5) has been transformed into

$\frac{d X}{d t}=\varepsilon \tilde{C}(t) g(X), \quad \grave{C}(t) \equiv \frac{1}{C(\hat{\mathrm{E}}[X(t)])}$,

where $\hat{E}[X(t)]$ expresses a certain typical value of the solution $X(t)$. Equation (19) is a differential equation driven by a temporally random noise $\tilde{C}(t)$, which does not have such a special feature as Eq.(5).

By use of the Markov approximation method [1], the transition probability density function of the solution $X(t)$ of Eq.(19) can be obtained as a solution of the generalized Fokker-Planck equation. Moreover, by neglecting a probability that the crack length 
decreases, the residual life distribution function has been obtained in an analytical form $[2]$ as

$H\left(t \mid x_{0}, x_{c}\right)=\bar{\Phi}\left[\frac{\mu_{Z}\left(x_{c} ; x_{0}\right)-\varepsilon \tilde{M}_{c} t}{\sqrt{2 G(t)}}\right]$,

$G(t)=\frac{\varepsilon \tilde{\sigma}_{C}^{2} \xi_{0}}{\tilde{M}_{C}} \int_{0}^{t} \frac{d t^{\prime}}{g\left(\hat{X}\left(t^{\prime}\right)\right)}$,

$\tilde{M}_{C} \equiv \mathrm{E}[\tilde{C}(t)], \quad \tilde{\sigma}_{C}^{2} \equiv \operatorname{Var}[\tilde{C}(t)]$,

where $\hat{X}(t)$ is the solution of the following ordinary differential equation:

$\frac{d \hat{X}}{d t}=\varepsilon \hat{M}_{C} g(\hat{X}), \quad \hat{X}(0)=x_{0}$.

The constant $\xi_{0}$ corresponds to a spatial correlation distance of the random field $C(x)$.

\section{Numerical example}

In this section, we apply the result obtained in the preceding section to the random growth of an edge crack in an infinite plate under uniform tensile stressing, schematically illustrated in Fig.2. The result will be compared with TI-model through numerical calculations for the residual life distribution.

The geometrical factor $\alpha$ and the correcting function $f(x)$ appearing in Eq.(3) are given as $\alpha=1.12$ and $f(x)=1$, respectively, for the edge crack. The spatial correlation function $\rho_{C}(x)$ is assumed to be exponential, that is,

$\rho_{C}(x)=\exp \left(-\frac{|x|}{L_{C}}\right)$

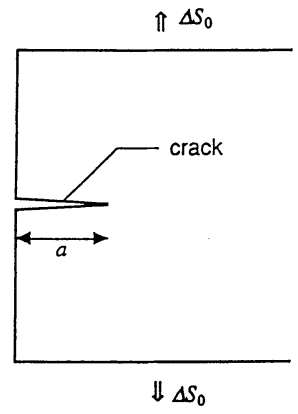

Fig.2 An edge crack in an infinite plate.

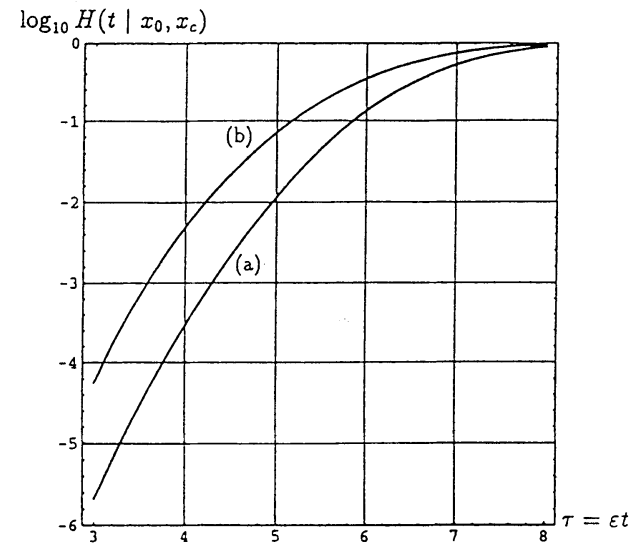

Fig.3 Residual life distributions by (a) the present method and (b) TI-model. $\left(x_{0}=0.05, x_{c}=1.0, m_{\imath}=3.0, \sigma_{C}=0.3, L_{C}=\xi_{0}=0.03\right)$ 
in which the constant $L_{C}$ represents the spatial correlation distance of $C(x)$, which is assumed here to be equal to the constant $\xi_{0}$ in TI-model.

Although $1 / C(x)$ does not generally follow the same distribution as $C(x)$, if we can assume that the probability distribution of $C(x)$ is approximated by a log-normal distribution, $1 / C(x)$ obeys the log-normal distribution with slightly different mean and variance as

$\mathrm{E}[1 / C(x)]=1+\sigma_{C}^{2}, \quad \operatorname{Var}[1 / C(x)]=\sigma_{C}^{2}\left(1+\sigma_{C}^{2}\right)^{2}$,

under the condition that the mean and variance of $C(x)$ are given by Eqs.(6) and (7),respectively. In what follows, we make a brief comparison between the present method and TI-model provided that Eq.(25) holds.

Figures $3 \sim 5$ show the residual life distributions as a function of $\tau \equiv \varepsilon t$ calculated by (a) the present method (given by Eq.(16)) and (b) TI-model (given by Eq.(20)), in which the vertical axes are plotted on logarithmic scale to show the precise behaviors of the so-called high-reliability region. In each figure, the parameter values of $x_{0}, x_{c}$ and $m$ are fixed to be $x_{0}=0.05, x_{c}=1.0$ and $m=3.0$, respectively, and those of $\sigma_{C}$ and $L_{C}$ are chosen as in the figures. The parameter values for Fig.5 are selected in such a way that the quantity $\tilde{\sigma}_{C}^{2}=\sigma_{C}{ }^{2}\left(1+\sigma_{C}{ }^{2}\right)^{2} \xi_{0}$ takes on the same value as that for Fig.3. In other word, with parameter values thus chosen, the uncertainty factor in TI-model becomes nearly the same in both cases.

From these figures we can observe that the residual life distribution for the present method shifts to a longer side in comparison to that for TI-model. This might be caused by the approximations introduced in the present model such that (1) the distribution of $Z\left(x ; x_{0}\right)$ is approximated to be Gaussian, and (2) the probability that $Z\left(x ; x_{0}\right)$ decreases is neglected in the first passage problem of $Z\left(x ; x_{0}\right)$.

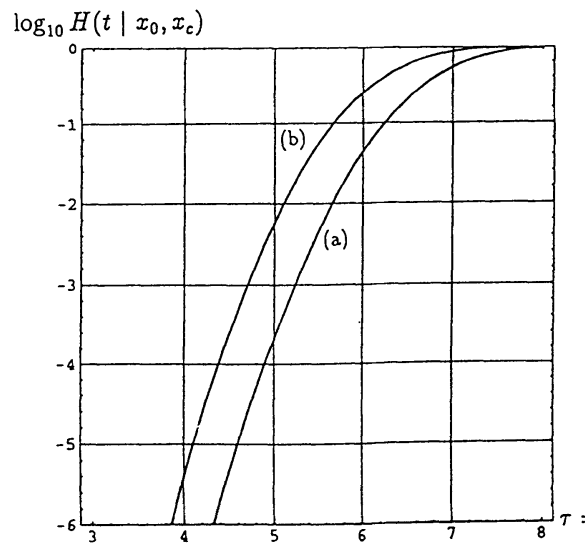

Fig.4 Residual life distributions by (a) the present method and (b) TI-model. $\left(x_{0}=0.05, x_{c}=1.0, m=3.0, \sigma_{C}=0.3, L_{C}=\xi_{0}=0.01\right)\left(x_{0}=0.05, x_{c}=1.0, m=3.0, \sigma_{C}=0.2, L_{C}=\xi_{0}=0.074\right)$

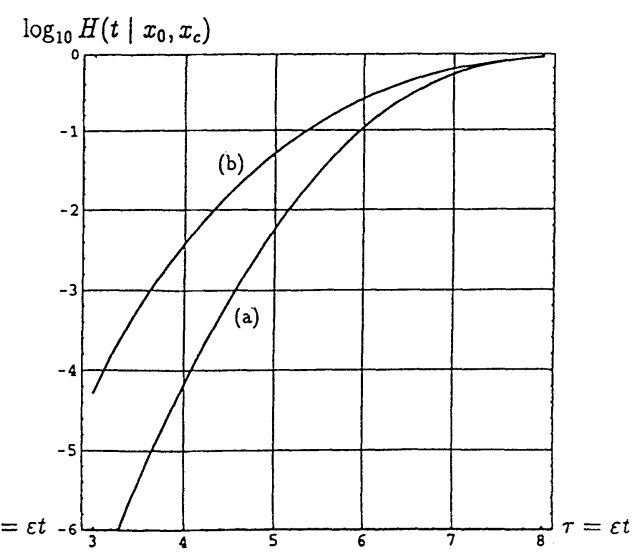

Fig.5 Residual life distributions by (a) the present method and (b) TI-model. 
However, in TI-model the approximations are also adopted such that (1) the introduced shape of the correlation function of $\tilde{C}(t)$ is not theoretical but is assumed, and (2) the decrease probability is also neglected in the first passage problem of $X(t)$. In this respect, it is impossible to determine which model gives a closer value to the true value. We have no other way but to utilize simulation techniques to prove this issue.

We can also observe that the difference in the result between these two models becomes a little larger when the values of $\sigma_{C}$ and $\xi_{0}$ become larger. In fact, when $\sigma_{C}$ assumes a value of nearly 0.1 , the difference becomes very small, which is unfortunately not shown in the figure for simplicity. Further, judging from the comparison between Figs. 3 and 5, TI-model reduces to neary the same result as long as the quantity $\tilde{\sigma}_{C}^{2} \xi_{0}$ takes on the same value. On the contrary, the present model produces a little difference.

\section{Concluding remarks}

In this paper, we have constructed a new mathematical model to describe the random fatigue crack growth by modeling the propagation resistance as a random field.

Although our present method can reflect the more precise engineering reality compared with TI-model, the more difficulties arise in solving the mathematical equations. However, these difficulties can be alleviated by use of the Markov approximation method, and we can derive the residual life distribution as well as the probability distribution of the crack length. In future studies, the accuracy of the approximation should be verified through computer simulation procedure.

\section{References}

1. A. Tsurui and H. Ishikawa, Application of Fokker-Planck Equation to a Stochastic Fatigue Crack Growth Model, Structural Safety, Vol. 4, pp. 15-29 (1986).

2. H. Ishikawa and A. Tsurui, A Stochastic Model of Fatigue Crack Growth in Consideration of Random Propagation Resistance, Trans. of JSME, Ser. A, Vol. 50, pp. 1309-1315 (1984) (in Japanese).

3. K. Sobczyk, Modeling of Random Fatigue Crack Growth, Engineering Fracture Mechanics, Vol. 26, pp. 609-623 (1986).

4. H. Itagaki, T. Ishizuka, T. Seki and M. Fujinami, Effect of Load Profile on Fatigue Crack Propagation under Narrow Band Random Loading, Proc. of JCOSSAR'91, pp. 411-418 (1991) (in Japanese).

5. T. Sasaki, S. Sakai and H. Okamura, Stochastic Modeling of Fatigue Crack Growth and its Numerical Analysis, Proc. of JCOSSAR'91, pp. 575-578 (1991) (in Japanese).

6. H. Tanaka and A. Tsurui, A Random Differential Equation of Special Type Defined on a Random Field and Its Application to Stochastic Crack Growth, Proc. of 12th Symposium on Reliability of Materials and Structures (JSMS), pp. 113-118 (1993) (in Japanese).

7. O. Ditlevsen, Random Fatigue Crack Growth - A First Passage Problem -, Engineering Fracture Mechanics, Vol. 23, No. 2, pp. 467-477 (1986). 